\title{
EDITORIAL
}

\section{The role of Case Reports in a medical journal}

Enoka Corea (Dhttp://orcid.org/0000-0003-1450-2098, Himani Molligoda (Dhttp://orcid.org/0000-0002-9216-9035,

Co-Editors

\section{Keywords: case reports}

This is an open-access article distributed under a Creative Commons Attribution-Share Alike 4.0 International License (CC BY-SA 4.0), which permits unrestricted use, distribution, and reproduction in any medium, provided the original author and source are attributed and materials are shared under the same license.

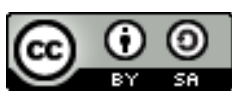

'Evidence-based medicine' has become the basis and the mantra of clinical management. Case reports, detailed descriptions of individual patients, are often looked down upon as being the 'weakest' form of evidence in a hierarchy that esteems meta-analysis and randomized clinical trials. It is stated that the knowledge put forward in a case report is anecdotal and not generalizable and features events rarely seen in clinical practice. However, it is important to understand that all forms of scientific communication, including case reports have a role to play in the compilation of medical evidence.

Case reports may, in the first instance, have an educational purpose. Clinicians learn best from anecdotes and through patient-based learning, and case reports narrate a clinical story. It is easier to remember the presentation, differential diagnosis, investigation, natural history and treatment of a disease when it is presented in the form of a real life patient narrative. The case should be illustrative and the discussion identify the features that are typical or not. However, many journals do not publish such case reports as they do not add to the current understanding of the disease. On the other hand, the advantage of patient narratives is that other aspects of clinical care, such as ethics, communication and doctor-patient relationship can be included. Educational case reports are most useful when the disease is rare and experience in that area is limited [1].

Case reports may also demonstrate an unusual aetiology for a clinical presentation, unusual presentation of a common disease, a clinical puzzle, unfamiliar use of diagnostic tests, novel methods of management, previously unreported adverse events or some other aspect that make the case unique and worthy of communication. Most importantly, the case report should convey a definite take home message and identify clearly how the knowledge gained from the case impacts on clinical practice [2].

Lastly, case reports may serve to generate initial hypotheses with regard to causation, investigation, management or prognosis of disease for verification or refutation in subsequent long-term formal studies. This may include the first identification of a new disease or first description of a new diagnostic or therapeutic method. The scientific basis for such a hypothesis and recommendations for future studies may be included in the case report, while clearly stating that evidence from the single case cannot be generalized. Case reports, therefore, may be the first inkling of important new knowledge and play a complementary role to clinical trials in the compilation of medical evidence [3]. 
The vast dossier of clinical case reports published in the public domain serve as a compendium of clinical information and data that can be mined to verify observations, extract trends and suggest theories of causation. To serve a useful purpose, case reports must have a strong rationale, be well written and focused and make clear to the reader why the case is important in the context of existing knowledge. The CARE guidelines, which include a checklist and template, set the standards for accuracy and completeness in reporting a case [4].

Case reports often represent the first attempt of a medical professional in scientific writing. Writing a case report is doubly educational in that it forces the author to conduct a comprehensive and up to date literature search on the topic and to reflect on the individual case. It necessitates the author to construct a detailed and comprehensive yet concise account of the case, highlighting its unique aspects vis a vis previous reports. The JPGIM is willing to support and facilitate this attempt by working with authors to improve the article to achieve publication standards.

\section{References}

1. Sun GH, Aliu O, Hayward RA. Open-access electronic case report journals: the rationale for case report guidelines. Journal of clinical epidemiology. 2013 Oct;66(10):1065. https://doi.org/10.1016/j.jclinepi.2013.04.001

2. Sun Z. Tips for writing a case report for the novice author. Journal of medical radiation sciences. 2013 Sep;60(3):108-13. https://doi.org/10.1002/jmrs.18

3. Agha R, Rosin RD. Time for a new approach to case reports. International journal of surgery case reports. 2010 Jan 1;1(1):1-3. https://doi.org/10.1016/j.ijscr.2010.04.001

4. Gagnier J, Kienle G, Altman DG, Moher D, Sox H, Riley D. The CARE guidelines: Consensus-based Clinical Case Reporting Guideline Development. Global advances in health and medicine, 2(5), 38-43. https://doi.org/10.7453/gahmj.2013.008 https://www.ncbi.nlm.nih.gov/pmc/articles/PMC3833570/ Accessed 5 ${ }^{\text {th }}$ March 2019 